\title{
Subthreshold microsecond laser for proliferative diabetic retinopathy: a randomized pilot study
}

This article was published in the following Dove Press journal:

Clinical Ophthalmology

\author{
Mahima Jhingan' \\ Abhilash Goud' \\ Hari Kumar Peguda' \\ Mitali Khodani' \\ Jeffrey K Luttrull ${ }^{2}$ \\ Jay Chhablani' \\ 'L V Prasad Eye Institute, Kallam Anji \\ Reddy Campus, Hyderabad, India; \\ ${ }^{2}$ Ventura County Retina Vitreous \\ Medical Group, CA, USA
}

\begin{abstract}
Aim: To compare the outcomes of subthreshold microsecond (STM) and continuous-wave laser (CWL) panretinal photocoagulation (PRP).

Methods: In this randomized, prospective, pilot study, 20 eyes of 10 subjects with symmetric severe non-proliferative (NPDR) or low-risk proliferative diabetic retinopathy (PDR) were included. Each eye of the subject was randomized into either CWL or STM PRP group. Patients were evaluated at baseline and at months 3,6, and 9 with color fundus photographs and visual field tests at each visit; however, electroretinography (ERG) was conducted at baseline and at month 9 . The primary outcome measure was the difference in disease progression between the groups. Secondary outcome measures included change in visual acuity, contrast visual acuity, retinal sensitivity on visual field test, and change in ERG parameters.
\end{abstract}

Results: During the 9-month follow-up, one eye of the STM group progressed to vitreous hemorrhage at the month 6 follow-up and required rescue conventional laser. The CWL group showed a drop in low-contrast visual acuity, visual field index, and scotopic b/a ratio in comparison to the STM group, although the difference was statistically insignificant $(p>0.05)$.

Conclusion: This prospective pilot study proposes microsecond PRP is non-inferior to CWL PRP and could be an alternative to CWL PRP to avoid associated complications in cases of severe NPDR and early PDR.

Keywords: panretinal photocoagulation, diabetic retinopathy, proliferative diabetic retinopathy, micropulse laser, microsecond laser

\section{Introduction}

Laser photocoagulation for the management of proliferative diabetic retinopathy (PDR) was introduced by Beetham et $\mathrm{al}^{1}$ in 1969 with the aid of ruby red laser. However, Zweng et $\mathrm{al}^{2}$ in 1971 first described the usage of argon laser for the management of PDR. It was not until the results of the Diabetic Retinopathy Study (DRS) ${ }^{3}$ and the Early treatment of Diabetic Retinopathy Study (ETDRS) 4 that the role of panretinal photocoagulation (PRP) was established as the gold standard treatment in preventing progression of complications of proliferative diabetic retinopathy (PDR). However, PRP has inherent adverse treatment effects. These include reduction in color and contrast sensitivity, loss of visual field and night vision, loss of visual acuity due to causation or exacerbation of macular edema, increased fibrovascular traction, and exudative choroidal detachment. ${ }^{5}$

With the description of PASCAL ${ }^{\circledR}$ or patterned scan laser by Blumenkranz et $\mathrm{al}^{6}$ in 2008, a semi-automated pattern allowing rapid application of multiple laser spots on the retina with a shorter pulse duration of 10-30 ms was possible. This modality reduced treatment duration and pain for the patient. Moreover, it allowed precise placement of spots with reduction in the number and size of visual field defects.
L V Prasad Eye Institute, Kallam Anji

Reddy Campus, Hyderabad 500034, India

Tel $+9 \mid 40306$ I2607

Fax +91 402354827 I

Email jay.chhablani@gmail.com 
Further, PASCAL ${ }^{\circledR}$ delivered less severe threshold burns than conventional longer pulse laser photocoagulation, reducing the severity of adverse treatment effects.

Pulsed subthreshold micropulse laser or microsecond laser (microsecond for NAVILAS ${ }^{\circledR}$ and micropulse for Iridex) is now commonly used in conditions such as diabetic macular edema and central serous chorioretinopathy, with good anatomical and visual outcomes, including improvement in functional vision. ${ }^{7}$ Pulsed subthreshold microsecond laser low-intensity/high-density subthreshold microsecond (STM) laser for PRP for proliferative diabetic retinopathy was first described by Luttrull et $\mathrm{al}^{8}$ in 2007 . They reported a reduced rate of vitreous hemorrhage and need for vitrectomy in their patients over a mean follow-up of 12 months, with reduced incidences of treatment-related complications. They noted that retinal destruction as a visible endpoint of photocoagulation is not actually necessary to achieve therapeutic benefit in the treatment of retinal vascular disease. In contrast, subthreshold diode microsecond laser is believed to act solely at the level of the retinal pigment epithelium (RPE) by normalizing its function, rather than by destruction. This was an uncontrolled, retrospective, consecutive case series study, and did not include visual fields or electroretinography in analysis of outcomes of their microsecond PRP protocols.

In the present study, we undertook to compare the change in visual acuity, visual fields, low-contrast visual acuity (LCVA), and disease progression between continuous-wave laser (CWL; $532 \mathrm{~nm}$ ) PRP and STM (577 nm) PRP in two eyes of the same subject with symmetrical low-risk PDR or severe non-proliferative diabetic retinopathy (NPDR) stage.

\section{Methods}

A randomized, prospective, controlled trial was conducted on ten subjects with a diagnosis of naïve, symmetric, bilateral, severe NPDR or low-risk PDR who presented to LV Prasad Eye Institute, Kallam Anji Reddy Campus, Hyderabad, India, between November 2014 and July 2015. Written informed consent was obtained from all subjects. The Institutional Review Board, Hyderabad Research Forum, India, approved the study, and all procedures adhered to the tenets of the Declaration of Helsinki. The chart review followed previously specified guidelines, as described earlier. ${ }^{9}$

All patients (age $\geq 18$ years) with type 1 or 2 diabetes with naïve, severe NPDR (SNPDR) or low-risk PDR (symmetric in both eyes) were identified. Diagnosis was made on the basis of the Diabetic Retinopathy Study Research Group. ${ }^{3}$ Exclusion criteria included patients with advanced PDR; history of prior laser treatment or vitrectomy in the study eye; history of anti-vascular endothelial growth factor (VEGF) injections received within 2 months; media opacities such as significant cataract, corneal opacity, or vitreous hemorrhage obscuring fundus details; history of cardiac event or cerebrovascular accident; coagulation abnormalities or use of anticoagulant other than aspirin, and conditions that affect documentation. Patients were not evaluated or treated for obstructive sleep apnea. Data collected at the initial visit included demographics, best-corrected visual acuity (BCVA) using ETDRS visual acuity, LCVA using COMPLOG, color fundus photographs (seven fields), infrared images (seven fields), autofluorescence images (seven fields), HVF 30-2, and Flash ERG.

A comprehensive ocular examination was carried out in all cases by the consultant in charge of the study (JC). Ocular investigations included optical coherence tomography (OCT) and fluorescein angiography (FFA), wherever indicated. FFA as well as fundus photography was undertaken using FF450plus Fundus Camera with VISUPAC (Carl Zeiss Meditec, Jena, Germany). OCT was done using Stratus OCT (Carl Zeiss Meditec, Jena, Germany), RTVue-100 (Optovue, Fremont, CA, USA), and Cirrus HD-OCT imaging systems (Carl Zeiss Meditec, Jena, Germany).

Both eyes with symmetric DR of one patient were randomized into two study groups using simple randomization by the simple method of flipping a coin to randomize each into either the CWL or STM group - one eye received CWL PRP on PASCAL ${ }^{\circledR} 532 \mathrm{~nm}$ (OptiMedica, Silicon Valley, USA) and the other eye received STM on Navilas ${ }^{\circledR} 577 \mathrm{~nm}$ (OD-OS GmbH, Teltow, Germany).

Parameters for treatment by the two machines are shown in Table 1.

Each eye completed PRP in one sitting on the same day. All eyes were followed within 1 hour as well as 1 month

Table I Laser parameters of two devices for panretinal photocoagulation

\begin{tabular}{|c|c|c|}
\hline & $\begin{array}{l}\text { Navilas }^{\circledR} \text { panretinal } \\
\text { microsecond pattern } \\
\text { laser }\end{array}$ & $\begin{array}{l}\text { Continuous-wave } \\
\text { PASCAL }{ }^{\circledR} \text { pattern } \\
\text { laser }\end{array}$ \\
\hline Wavelength (nm) & 577 & 532 \\
\hline Laser & Microsecond & $\begin{array}{l}\text { Conventional } \\
\text { (continuous wave) }\end{array}$ \\
\hline Power & $\begin{array}{l}15 \% \text { DC with } 20 \% \text { addition } \\
\text { to the test burn power }\end{array}$ & To achieve white burn \\
\hline Pulse duration & $200 \mathrm{~ms}$ (envelope) & $30 \mathrm{~ms}$ \\
\hline Spot size & 300 microns & $200-400$ microns \\
\hline Pattern spacing & Confluent & I.5-burn apart \\
\hline $\begin{array}{l}\text { Total number } \\
\text { spots per session }\end{array}$ & $\begin{array}{l}4,000-6,000 \text { spots in four } \\
\text { quadrants ( } 2-3 \text { sittings if } \\
\text { required, can be conducted } \\
\text { on consecutive days) }\end{array}$ & $\begin{array}{l}2,000 \text { spots in four } \\
\text { quadrants ( } 2-3 \text { sittings) }\end{array}$ \\
\hline
\end{tabular}


after treatment, and a full-field color image (seven fields) was obtained to identify any "late blooming" threshold laser lesions that were invisible at the time of treatment. At all follow-up visits on months 3, 6, and 9, color fundus photographs (seven fields), infrared images (seven fields), autofluorescence images (seven fields), and HVF 30-2 were done. Flash ERG was performed at baseline and at month 9. These were evaluated by the consultant, JC, and then correlated with findings at presentation.

Rescue CWL PASCAL ${ }^{\circledR}$ PRP was offered if progression of PDR was noted as defined - as an occurrence of vitreous hemorrhage or development of neovascular complexes.

All patients as well as the trained optometrist who assessed visual acuity, contrast visual acuity, and visual fields were masked to randomization.

\section{Outcome measures}

Primary outcome measure: The difference in disease progression between the groups was the primary outcome measure.

Secondary outcome measures: These included change in visual acuity, contrast visual acuity, retinal sensitivity on HVF 30-2, and change in ERG parameters.

Statistical analysis was conducted using SPSS v. 16.0 (SPSS, Chicago, IL, USA), with special emphasis on the aforementioned information. $p$-values less than 0.05 were considered as indicative of statistical significance.

\section{Results}

Twenty eyes of ten patients with symmetric SNPDR (four eyes) or LRPDR (16 eyes) were included in this study, with the two eyes of each individual being randomized into CWL PRP or STM PRP, respectively. Every patient had symmetric grade of diabetic retinopathy.

The average age of study patients was $53 \pm 5.6$ years, with seven males in the study. At baseline, lens findings were similar in both the laser groups. The distribution of DR class was equal in both groups - each having three eyes with SNPDR, and seven with LRPDR. No eye in either group had diabetic macular edema. The average central macular thicknesses were $274 \pm 56$ and $282 \pm 32 \mu \mathrm{m}$ in the CWL and STM groups, respectively.

Changes in various parameters at month 9 are shown in Table 2.

\section{Disease progression}

During the 9-month follow-up, in the STM group, one eye demonstrated clinical progressive DR and one eye progressed to transient vitreous hemorrhage 6 months after treatment,
Table 2 Comparison of various outcome measures between the two groups

\begin{tabular}{llllll}
\hline & \multicolumn{2}{l}{ Conventional, Mean $( \pm$ SD $)$} & & Microsecond, Mean $( \pm$ SD $)$ \\
\cline { 2 - 3 } \cline { 5 - 6 } & Baseline & Last visit & & Baseline & Last visit \\
\hline BCVA & $0.15( \pm 0.15)$ & $0.21( \pm 0.21)$ & $0.13( \pm 0.17)$ & $0.17( \pm 0.1 \mathrm{I})$ \\
(logMAR) & & $(p=0.55)$ & & $(p=0.53)$ \\
ETDRS & $77.00( \pm 8.70)$ & $74.33( \pm 10.45)$ & $78.50( \pm 8.05)$ & $76.33( \pm 6.40)$ \\
(letters) & & $(p=0.56)$ & & $(p=0.53)$ \\
LCVA & $0.36( \pm 0.28)$ & $0.39( \pm 0.3 \mathrm{I})$ & $0.36( \pm 0.20)$ & $0.35( \pm 0.20)$ \\
& & $(p=0.83)$ & & $(p=0.88)$ \\
VFI & $0.92( \pm 0.07)$ & $0.885( \pm 0.06)$ & $0.95( \pm 0.04)$ & $0.92( \pm 0.04)$ \\
& & $(p=0.299)$ & & $(p=0.24)$ \\
Scotopic & $2.32( \pm 0.39)$ & $2.38( \pm 0.47)$ & $2.18( \pm 0.52)$ & $2.06( \pm 0.59)$ \\
b/a ratio & & $(p=0.83)$ & & $(p=0.74)$ \\
Photopic & $4.24( \pm 0.98)$ & $4.9( \pm 1.56)$ & $4.12( \pm 1.28)$ & $4.52( \pm 1.49)$ \\
b/a ratio & & $(p=0.44)$ & & $(p=0.66)$ \\
\hline
\end{tabular}

Abbreviations: BCVA, best-corrected visual acuity; ETDRS, Early Treatment of Diabetic Retinopathy Study; LCVA, low-contrast visual acuity; VFI, visual field index.

for which rescue PRP was done; no clinical neovascular complex was noted in this patient. None of the eyes had any notable adverse treatment effect or progression in fibrovascular traction during follow-up. Figure 1 shows a representative case.

\section{Visual acuity}

The mean $\log$ MAR BCVA at presentation in both the groups was comparable, with a value of $77 \pm 8.7$ letters (20/30 Snellen's equivalent) in the CWL group and 78.5 \pm 8 letters (20/30 Snellen's equivalent) in the STM group.

This dropped to $74.3 \pm 10.45$ letters (20/30 Snellen's equivalent) in the CWL group and $76.3 \pm 6.4$ letters $(20 / 30$ Snellen's equivalent) in the STM laser group. This drop of three letters on the ETDRS chart in the CWL group versus three letters in the STM group was found to be insignificant for both the groups with a $p$-value of 0.55 and 0.53 , respectively, for both the groups.

\section{Low-contrast visual acuity}

The LCVA dropped from $0.36 \pm 0.28$ (20/45 Snellen equivalent) $\log$ MAR units to $0.39 \pm 0.31$ units (20/50 Snellen equivalent) in the CWL group ( $p=0.83$ ). In the STM group, the LCVA improved slightly, from a pre-laser LCVA of $0.36 \pm 0.2$ $\operatorname{logMAR}(20 / 50$ Snellen equivalent) to a post-laser LCVA of $0.35 \pm 0.2$ (20/45 Snellen's equivalent; $p=0.88$ ).

\section{Visual field parameters}

The visual field index (VFI) in the CWL group dropped from a mean of $0.92 \pm 0.074$ at presentation to $0.85 \pm 0.056$ $(p=0.29)$, whereas the VFI in the STM group dropped 

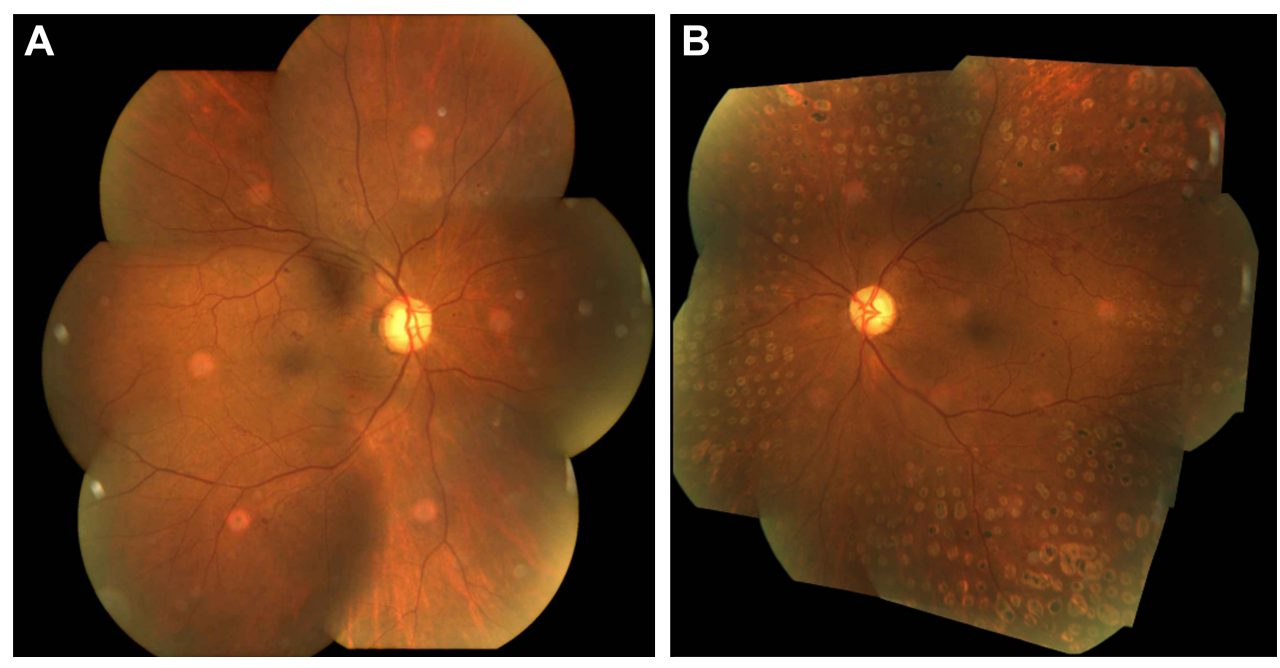

Figure I A 62-year old man diagnosed with low-risk proliferative diabetic retinopathy underwent right-eye (A) microsecond panretinal photocoagulation and conventional continuous-wave laser panretinal photocoagulation in his left eye (B). At the 9-month follow-up, the right eye does not show any scarring, with no progression of the disease. The left eye shows laser scars with stable retinal status.

from $0.945 \pm 0.037$ at presentation to $0.92 \pm 0.04$ at the last visit $(p=0.62)$.

\section{Electroretinography}

The scotopic and photopic b/a ratios were statistically unchanged following treatment in both groups. At presentation, the scotopic b/a was $2.32 \pm 0.38$ and $2.18 \pm 0.51$ in the CWL and STM groups, respectively. This changed to a value of $2.38 \pm 0.47(p=0.83)$ and $2.06 \pm 0.59(p=0.74)$ in the CWL and STM groups, respectively. The photopic b/a ratio, on the other hand, showed an improvement in both the CWL and the STM groups. The pre-laser values changed in the CWL group from $4.24 \pm 0.98$ to $4.9 \pm 1.55$ ( $p=0.44$ ). Similarly, in the STM group, the values changed from a pre-laser value of $4.12 \pm 1.28$ to a post-laser value of $4.52 \pm 1.59(p=0.66)$.

Between-group comparison of various outcome measures are shown in Table 2.

\section{Discussion}

Our study showed that only one eye in the STM group, which had severe NPDR at baseline, progressed to vitreous hemorrhage at month 6. Moreover, it reported no significant difference between various parameters, such as visual acuity, LCVA, or visual field, between the CWL and STM groups. There was a slight advantage noted in the STM group in terms of LCVA as well as visual field. The CWL group showed a drop in the LCVA, visual field index, and scotopic b/a ratio in comparison to the STM group, although the change was statistically insignificant.

Our study raises an important concern about the conventional belief that retinal destruction is required to control the neovascularization process. We noticed clinical progression of neovascularization in one eye (10\%) in the STM group. Although the eye with SNPDR developed a hemorrhage, no clinically detectable new lesions were seen, and even though we have treated this as a progression as per the definition in our study, we feel that, owing to lack of wide-field imaging, a neovascular complex in the far periphery could have been missed at diagnosis as well at further follow-ups or it could have been induced by posterior vitreous detachment. While resolution of neovascularization is the conventional treatment goal of panretinal laser for PDR, arrest and regression is the norm, and complete disappearance is rare with conventional photocoagulation and long-term anti-VEGF suppressive treatment. Echoing the findings of Luttrull et al, ${ }^{8}$ eliminating (STM) or reducing (PASCAL CWL) retinal damage - and, thus, inflammation - appeared to minimize preretinal fibrosis and contraction. This may generally lead to a more benign postoperative clinical course.

An advantage of microsecond laser over the conventional laser PRP includes no pain during treatment. Microsecond PRP can be repeated any number of times without adverse treatment effects. In this study, eyes were treated once at study entry. It is possible that periodic retreatment, permitted by treatment safety, may improve treatment results. Due to the lack of treatment-associated inflammation, microsecond PRP might be advantageous in eyes with extensive neovascularization and a thickened or partially detached hyaloid, which is at higher risk of tractional detachment following conventional laser PRP.

Due to ethical concerns, we treated eyes with significant, but not high-risk, DR, as there is no generally accepted 
need or mode of treatment at this stage of the disease that can be considered, while somewhat late in the course, as being "preventative." The lack of generally accepted interventions for SNPDR and LRDR reflect adverse treatment effects associated with conventional PRP, which weigh against the benefits of early treatment and has been, thus far, the only available mode of treatment. While ranibizumab has been approved recently by the US FDA for treatment of diabetic retinopathy, long-term use of an expensive, short-acting, intravitreal medication for prevention of a chronic progressive disease is inherently problematic on many levels. While we report no significant improvements in secondary outcome measures, we also report no significant worsening. It must be remembered that the idea of preventive treatment changes nothing except the likelihood of disease progression. In this respect, both STM and CWL panretinal laser were found to stop progression of retinopathy without adverse treatment effects. ${ }^{10}$ Thus, both may offer a more useful mode of preventive treatment in DR than conventional PRP or intravitreal drug injection. Further study is clearly necessary in this regard. However, we believe early treatment of SNPDR and LRPDR by STM or CWL panretinal laser may be useful in selected patients. While equally effective in this small study, because of that specific equality, we believe STM is to be preferred on the basis of its superior safety (no laser-induced retinal damage), comfort, and repeatability.

Limitations of our study include a small sample size and short follow-up. Due to the unavailability of widefield imaging, we are unable to comment on the changes in peripheral neovascularization or ischemic areas during follow-up visits in both groups. We are unable to comment on subthreshold PRP in eyes with high-risk PDR, as we could not include this group due to ethical concerns. Strengths of our study include the evaluation of two laser modalities in the same subject, which excludes the bias caused by the systemic status of the subject. We included patients with symmetric disease to avoid any selection bias. In our study, patients as well as the evaluating optometrist were masked to the randomization.

In conclusion, our prospective pilot study proposes microsecond PRP is non-inferior to CWL PRP and could be an alternative to conventional CWL PRP in severe NPDR and early PDR to avoid complications associated with CW laser. A prospective study with a larger sample size with longer follow-up is warranted to establish the role of microsecond laser for PRP.

\section{Disclosure}

The authors report no conflicts of interest in this work.

\section{References}

1. Beetham WP, Aiello LM, Balodimos MC, Koncz L. Ruby-laser photocoagulation of early diabetic neovascular retinopathy: preliminary report of a long-term controlled study. Trans Am Ophthalmol Soc. 1969; 67:39-67.

2. Zweng HC, Little HL, Peabody RR. Argon laser photocoagulation of diabetic retinopathy. Arch Ophthalmol. 1971;86(4):395-400.

3. The Diabetic Retinopathy Study Research Group. Photocoagulation treatment of proliferative diabetic retinopathy: the second report of diabetic retinopathy study findings. Ophthalmology. 1978;85(1):82-106.

4. Early photocoagulation for diabetic retinopathy. ETDRS report number 9. Early Treatment Diabetic Retinopathy Study Research Group. Ophthalmology. 1991;98(5 Suppl):766-785.

5. Liang JC, Huamonte FU. Reduction of immediate complications after panretinal photocoagulation. Retina. 1984;4(3):166-170.

6. Blumenkranz MS, Yellachich D, Andersen DE, et al. Semiautomated patterned scanning laser for retinal photocoagulation. Retina. 2006; 26(3):370-376.

7. Kozak I, Luttrull JK. Modern retinal laser therapy. Saudi J Ophthalmol. 2015;29(2):137-146.

8. Luttrull JK, Musch DC, Spink CA. Subthreshold diode micropulse panretinal photocoagulation for proliferative diabetic retinopathy. Eye (Lond). 2008;22(5):607-612.

9. Gilbert EH, Lowenstein SR, Koziol-McLain J, Barta DC, Steiner J. Chart reviews in emergency medicine research: Where are the methods? Ann Emerg Med. 1996;27(3):305-308.

10. Luttrull JK, Sinclair SH. Safety of transfoveal subthreshold diode micropulse laser for fovea-involving diabetic macular edema in eyes with good visual acuity. Retina. 2014;34(10):2010-2020.
Clinical Ophthalmology

\section{Publish your work in this journal}

Clinical Ophthalmology is an international, peer-reviewed journal covering all subspecialties within ophthalmology. Key topics include: Optometry; Visual science; Pharmacology and drug therapy in eye diseases; Basic Sciences; Primary and Secondary eye care; Patient Safety and Quality of Care Improvements. This journal is indexed on

\section{Dovepress}

PubMed Central and CAS, and is the official journal of The Society of Clinical Ophthalmology (SCO). The manuscript management system is completely online and includes a very quick and fair peer-review system, which is all easy to use. Visit http://www.dovepress.com/ testimonials.php to read real quotes from published authors. 